\title{
Increased levels of red cell distribution width is correlated with presence of left atrial stasis in patients with non-valvular atrial fibrillation
}

\author{
Adnan Kaya, ${ }^{1}$ Ceyhan Tukkan, ${ }^{2}$ Ahmet Taha Alper, ${ }^{2}$ Baris Gungor, ${ }^{2}$ \\ Kazim Serhan Ozcan, ${ }^{3}$ Mustafa Adem Tatlisu, ${ }^{2}$ Ahmet Ilker Tekkesin, ${ }^{2}$ \\ Fatma Ozpamuk Karadeniz, ${ }^{4}$ Gulay Gok, ${ }^{2}$ Osman Kayapinar ${ }^{1}$ \\ ${ }^{1}$ Department of Cardiology, Duzce University Faculty of Medicine, Duzce, Turkey \\ 2Department of Cardiology, Dr. Siyami Ersek Cardiovacular Surgery Hospital, Istanbul, Turkey \\ 3Department of Cardiology, Derince Training and Research Hospital, Kocaeli, Turkey \\ ${ }^{4}$ Department of Cardiology, Erzurum Training and Research Hospital, Erzurum, Turkey
}

\begin{abstract}
OBJECTIVE: Red cell distribution width (RDW) and neutrophil to lymphocyte ratio (NLR) have been found to be associated with non-valvular atrial fibrillation (AF) and cardiovascular diseases. However, correlation of these parameters with presence of left atrial (LA) thrombus and/or spontaneous echo contrast (SEC) in patients with non-valvular AF has not been clarified. This study was an investigation of correlation of RDW, NLR, and clinical risk factors with LA thrombus and dense SEC in patients with non-valvular AF in the Turkish population.

METHODS: The demographic, laboratory, and echocardiographic properties of 619 non-valvular AF patients who underwent transesophageal echocardiography (TEE) examination before direct current cardioversion (DCCV) or $\mathrm{AF}$ ablation treatment were retrospectively investigated. Complete blood count $(\mathrm{CBC})$ and biochemical parameters were studied 6 to 12 hours before TEE examination. Left atrial stasis (LAS) markers were noted as presence of left atrial/left atrial appendage (LA/LAA) thrombus or dense spontaneous echo contrast (DSEC).

RESULTS: Total of 325 (52\%) patients with LAS were compared with 294 patients (48\%) without LAS. In the LAS group, there were $274(84 \%)$ patients with LA/LAA thrombus and $51(16 \%)$ patients with DSEC. LAS (+) group, values for RDW (14.85 \pm 1.48 vs. $13.77 \pm 1.30 ; p<0.01)$, NLR (2.38 [1.58], vs. 2.10 [1.35]; $\mathrm{p}<0.01)$ and C-reactive protein $(0.95[0.61]$ vs. $0.88[0.60] \mathrm{mg} / \mathrm{L} ; \mathrm{p}<0.01)$ were significantly higher than seen in LAS (-) group. In multivariate regression analysis, increased level of RDW, age, male gender, heart failure, duration of AF $>6$ months, and international normalized ratio $<2$ were independently correlated with presence of LAS.
\end{abstract}

CONCLUSION: Our study indicated that increased level of RDW is independently correlated with higher risk for development of LAS in patients with non-valvular AF.

Keywords: Left atrial thrombus; non-valvular atrial fibrillation; red cell distribution width.

Received: February 21, 2017 Accepted: April 12, 2017 Online: May 10, 2017

Correspondence: Dr. Adnan KAYA. Duzce Universitesi Tip Fakultesi, Kardiyoloji Anabilim Dali, Duzce, Turkey.

Tel: +90 380 - 5421416 e-mail: adnankaya@ymail.com

(c) Copyright 2017 by Istanbul Northern Anatolian Association of Public Hospitals-Available online at www.kuzeyklinikleri.com 
$\mathrm{A}$ trial fibrillation (AF) is the most common cardiac arrhythmia and is associated with increased cardiovascular risks [1]. The major complication of $\mathrm{AF}$ is formation of thrombus in the left atrium (LA) and/or left atrial appendage (LAA) causing thromboembolism. Abnormal flow dynamics in the LA and LAA cause blood stasis, endothelial damage, and thrombus formation, even in the absence of valvular disease [2]. The inflammatory process is important in pathogenesis of AF, which is associated with the stimulation of coagulation cascade and thrombus formation [3].

Recently, correlation of red cell distribution width (RDW) and neutrophil to lymphocyte ratio (NLR) with inflammatory status has been demonstrated [4]. In addition, prognostic importance of these parameters in various cardiovascular diseases, including non-valvular AF, has been found $[5,6]$. Transesophageal echocardiography (TEE) is the criterion standard for detection of LA/LAA thrombus and dense spontaneous echo contrast (DSEC). These findings indicate left atrial stasis (LAS), and are correlated with higher risk of thromboembolism during electrical cardioversion and $\mathrm{AF}$ ablation procedures [7-9].

The aim of this study was to investigate the correlation of pre-procedural RDW, NLR, and clinical risk factors with presence of LAS in patients with non-valvular AF who had undergone TEE examination.

\section{MATERIALS AND METHODS}

\section{Patient selection}

This study was a retrospective, cross-sectional study conducted between January 2009 and June 2014 at our tertiary cardiovascular hospital. Study consisted of 325 consecutive LAS (+) patients who had undergone TEE between January 2009 and June 2014 and randomly selected 294 LAS (-) patients from the same time interval before electrical cardioversion or AF ablation treatment. Patients older than 80 years of age, with valvular AF, post-operative AF, recent acute coronary syndrome, anemia (for men hemoglobin $<13 \mathrm{gr} /$ $\mathrm{dL}$, for women hemoglobin $<12 \mathrm{gr} / \mathrm{dL}$ ), acute or chronic inflammatory disease, renal failure, hepatic failure, malignancy, or with other indication of chronic anticoagulation or history of blood transfusion within 3 months were excluded. All patient risk factors, clinical information, laboratory and demographic data were obtained from hospital records.

\section{Echocardiography examination}

Transesophageal echocardiography was performed with GE Vivid 7 device (GE Healthcare, Inc. Chicago, IL, USA) and $6 \mathrm{~T}$ phased array multiplane transesophageal (2.9 to $7.0 \mathrm{MHz}$ ) probe. All examinations were performed after administration of local anesthesia of hypopharynx with lidocaine spray and sedation with 2 to $5 \mathrm{mg}$ intravenous administration of midazolam. LA and LAA were investigated for presence of thrombus and DSEC in different tomographic planes of TEE. LA/LAA thrombus was defined as (1) masses adhering to LA wall or LAA, (2) masses moving independently from the LAA wall, (3) masses of different echogenicity density from LAA wall, and (4) confirmation of mass in more than 1 imaging plane. Special efforts were made to discriminate pectinate muscles from thrombi. In case of doubt, images were re-evaluated until consensus was reached. Spontaneous echo contrast (SEC) scoring was done from $1+$ to $4+$ as described by Fatkin et al. [10]. Patients with 3+ and 4+ SEC were defined as DSEC. Patients with thrombus of LA/LAA and/or DSEC formed study group [LAS (+) group] and patients who did not have these findings formed control group [LAS (-) group]. Congestive heart failure (CHF) was defined as having diagnosis of ejection fraction preserved heart failure and/or heart failure with systolic dysfunction (ejection fraction less than 49\%) and use of heart failure medication. In addition, Congestive Heart Failure, Hypertension, Age, Diabetes Mellitus, Stroke $\left(\mathrm{CHADS}_{2}\right)$ score and Congestive Heart Failure, Hypertension, Age, Diabetes Mellitus, Stroke, Vascular Disease, Sex ( $\left.\mathrm{CHA}_{2} \mathrm{DS}_{2}-\mathrm{VASc}\right)$ score were calculated for each patient in order to evaluate risk of thromboembolism and stroke [11]. The study was approved by the local ethics committee of the hospital. 


\section{Laboratory analysis}

Fasting blood samples were drawn according to hospital protocol from antecubital vein puncture into ethylenediaminetetraacetic acid-treated or plain tubes on day of admission 6 to 12 hours before TEE examination. Complete blood count (CBC) testing utilized clinical laboratory methods (Coulter LH 780 Hematology Analyzer; Beckman Coulter Inc., Brea, CA) for hemoglobin, total white blood cell (WBC) count, platelet count and RDW. Baseline NLR was calculated by dividing neutrophil count by lymphocyte count. High-sensitivity $\mathrm{C}$-reactive protein (CRP) level was measured with Cobas Integra analyzer (Roche Diagnostics, Basel, Switzerland) using turbidimetric method.

\section{Statistical analysis}

Statistical analyses were performed using SPSS for Windows, Version 15.0 (SPSS Inc., Chicago, IL, USA) software. Continuous variables were checked for normal distribution assumption using Kolmogorov-Smirnov test and were reported as mean \pm SD or median (interquartile range). Differences between LAS (+) and (-) groups were evaluated using Student's t-test or Mann-Whitney U test. Categorical variables were tested with Pearson's chisquared test and Fisher's exact test. Receiver operating curves (ROC) were generated to define cut-off values (upper left corner of ROC as point of maximum sensitivity and specificity) of RDW for presence of LAS in the study population. In addition, univariate and multivariate binary logistic regression analysis was performed to investigate independent correlates of LAS. Variables with $p<0.10$ in univariate analysis were included in multivariate regression analysis. All $\mathrm{p}$ values were two-sided and $\mathrm{p}$ value $<0.05$ was considered statistically significant.

\section{RESULTS}

The study included 325 patients with LAS and 294 patients without LAS. In the LAS (+) group, there were $274(84 \%)$ patients with LA/LAA thrombus and 51 (16\%) patients with DSEC. Clinical and demographic properties of the study population are summarized in Table 1. Study patients were older
$(62.8 \pm 12.5$ vs. $54.5 \pm 13.9 ; p=0.01)$, and frequency of male gender was higher ( $63 \%$ vs. $54 \% ; p=0.03$ ) compared with control group. Frequency of patients with hypertension, diabetes mellitus, CHF, and vascular diseases was higher and duration of AF was longer in LAS (+) group. Mean $\mathrm{CHADS}_{2}$ score $(1.90 \pm 1.16$ vs. $1.08 \pm 1.01 ; \mathrm{p}<0.01)$ and mean $\mathrm{CHA}_{2} \mathrm{DS}_{2}-\mathrm{VASc}$ score $(2.43 \pm 1.40$ vs. $1.58 \pm 1.19 ; \mathrm{p}<0.01)$ were significantly higher in LAS $(+)$ group. In addition, percentage of patients with $\mathrm{CHADS}_{2} \geq 3, \mathrm{CHA}_{2} \mathrm{DS}_{2}-\mathrm{VASc} \geq 3$ was significantly higher in LAS $(+)$ group compared with LAS (-) group.

In total, 370 patients had warfarin treatment and rate of warfarin use was significantly higher in the LAS (+) group ( $82 \%$ vs. $36 \%$; $<<0.01)$. When only patients with warfarin treatment were considered, rate of LA/LAA thrombus was $66 \%$. Frequency of patients with optimal anticoagulation (international normalized ratio between 2-3) at time of TEE examination was $78 \%$. Only patients without LAS had undergone electrical cardioversion or AF ablation procedure and none of the treated patients had thromboembolic complication.

Hematological and biochemical parameters of study groups are compared in Table 2. Statistically significant difference was observed between creatinine levels, whereas hemoglobin level and WBC, platelet, neutrophil, and lymphocyte counts were not different between groups. In LAS (+) group, $\operatorname{NLR}(2.38$ [1.58] vs. 2.10 [1.35]; p<0.01), RDW $(14.85 \pm 1.48$ vs. $13.77 \pm 1.30 ; \mathrm{p}<0.01)$, and CRP (0.95 [0.61] vs. 0.88 [0.60] mg/L; p<0.01) levels were significantly higher compared with control group. In subgroup analysis, when patients with LA/LAA thrombus were compared with patients with DSEC, RDW $(14.84 \pm 1.56$ vs. $14.34 \pm 0.93$; $\mathrm{p}=0.32), \operatorname{NLR}(2.25[1.62]$ vs. 2.32 [0.93]; $\mathrm{p}=0.32)$, and CRP (0.96 [0.60] vs. $0.95[0.51]$ $\mathrm{mg} / \mathrm{L} ; \mathrm{p}=0.91$ ) levels were not significantly different (Figure 1).

In ROC curve analysis, area under curve (AUC) of RDW was significantly higher compared with AUC of NLR and CRP for discrimination of LAS in the study population ( 0.732 vs. 0.579 vs. 0.584 , respectively; $\mathrm{p}<0.01$ ) (Figure 2 ). Cut-off level of 
TABLE 1. Demographic characteristics and risk factor identification for left atrial stasis (+) and (-) groups

\begin{tabular}{|c|c|c|c|c|c|}
\hline \multirow{3}{*}{ Age, years } & \multicolumn{2}{|c|}{ LAS $(+)(n=325)$} & \multicolumn{2}{|c|}{ LAS $(-)(n=294)$} & \multirow[t]{2}{*}{$\mathrm{p}$} \\
\hline & $\mathrm{n}$ & $\%$ & $\mathrm{n}$ & $\%$ & \\
\hline & \multicolumn{2}{|c|}{$62.8 \pm 12.5$} & \multicolumn{2}{|c|}{$54.5 \pm 13.9$} & $<0.01$ \\
\hline Male sex & 206 & 63 & 161 & 54 & 0.03 \\
\hline Hypertension & 247 & 76 & 164 & 55 & $<0.01$ \\
\hline Diabetes mellitus & 49 & 15 & 23 & 8 & $<0.01$ \\
\hline Stroke & 24 & 7 & 14 & 5 & 0.24 \\
\hline Congestive heart failure & 59 & 18 & 12 & 4 & $<0.01$ \\
\hline Vascular disease & 57 & 17 & 19 & 6 & $<0.01$ \\
\hline AF duration $>6$ months & 278 & 85 & 143 & 49 & $<0.01$ \\
\hline LA/LAA thrombus & 274 & 84 & - & - & - \\
\hline DSEC & 51 & 16 & - & - & - \\
\hline $\mathrm{CHADS}_{2}$ score & \multicolumn{2}{|c|}{$1.90 \pm 1.16$} & \multicolumn{2}{|c|}{$1.08 \pm 1.01$} & $<0.01$ \\
\hline $\mathrm{CHADS}_{2}$ score $>3$ & 85 & 26 & 28 & 10 & $<0.01$ \\
\hline $\mathrm{CHA}_{2} \mathrm{DS}_{2}$-VASc score & \multicolumn{2}{|c|}{$2.43 \pm 1.40$} & \multicolumn{2}{|c|}{$1.58 \pm 1.19$} & $<0.01$ \\
\hline $\mathrm{CHA}_{2} \mathrm{DS}_{2}-\mathrm{VASC}>3$ & 148 & 45 & 59 & 20 & $<0.01$ \\
\hline \multicolumn{6}{|l|}{ Treatment } \\
\hline Warfarin & 265 & 82 & 105 & 36 & $<0.01$ \\
\hline Acetylsalicylic acid & 50 & 15 & 148 & 50 & $<0.01$ \\
\hline No treatment & 10 & 3 & 41 & 14 & \\
\hline
\end{tabular}

AF: Atrial fibrillation; LA: Left atrium; LAA: Left atrial appendage; DSEC: Dense spontaneous echo contrast; CHADS: Congestive Heart Failure, Hypertension, Age, Diabetes Mellitus, Stroke; $\mathrm{CHA}_{2} \mathrm{DS}$-VASc: Congestive Heart Failure, Hypertension, Age, Diabetes Mellitus, Stroke, Vascular Disease, Sex.

TABLE 2. Comparison of hematological parameters between left atrial stasis $(+)$ and $(-)$ groups

\begin{tabular}{lccc} 
& $\begin{array}{c}\text { LAS }(+) \\
(\mathrm{n}=325)\end{array}$ & $\begin{array}{c}\text { LAS }(-) \\
(\mathrm{n}=294)\end{array}$ & $\mathrm{p}$ \\
\hline Creatinine, $\mathrm{mg} / \mathrm{dL}$ & $0.93 \pm 0.25$ & $0.87 \pm 0.24$ & $<0.01$ \\
Hemoglobin, $\mathrm{g} / \mathrm{dL}$ & $13.8 \pm 1.5$ & $14.0 \pm 1.3$ & 0.21 \\
Platelet, $10^{3} / \mu \mathrm{L}$ & $246 \pm 75$ & $249 \pm 77$ & 0.56 \\
WBC, $10^{3} / \mu \mathrm{L}$ & $7.92 \pm 1.85$ & $7.91 \pm 2.23$ & 0.77 \\
Neutrophil, $10^{3} / \mu \mathrm{L}$ & $5.03 \pm 1.65$ & $4.79 \pm 1.79$ & 0.09 \\
Lymphocyte, $10^{3} / \mu \mathrm{L}$ & $2.12 \pm 1.12$ & $2.26 \pm 0.91$ & 0.10 \\
NLR & $2.38[1.58]$ & $2.10[1.35]$ & $<0.01$ \\
RDW, \% & $14.85 \pm 1.48$ & $13.77 \pm 1.30$ & $<0.01$ \\
CRP, mg/L* & $0.95[0.61]$ & $0.88[0.60]$ & $<0.01$ \\
\hline
\end{tabular}

CRP: C-reactive protein; NLR: Neutrophil to lymphocyte ratio; RDW: Red cell distribution width; WBC: White blood cell. *CRP level could be obtained in only 524 patients.

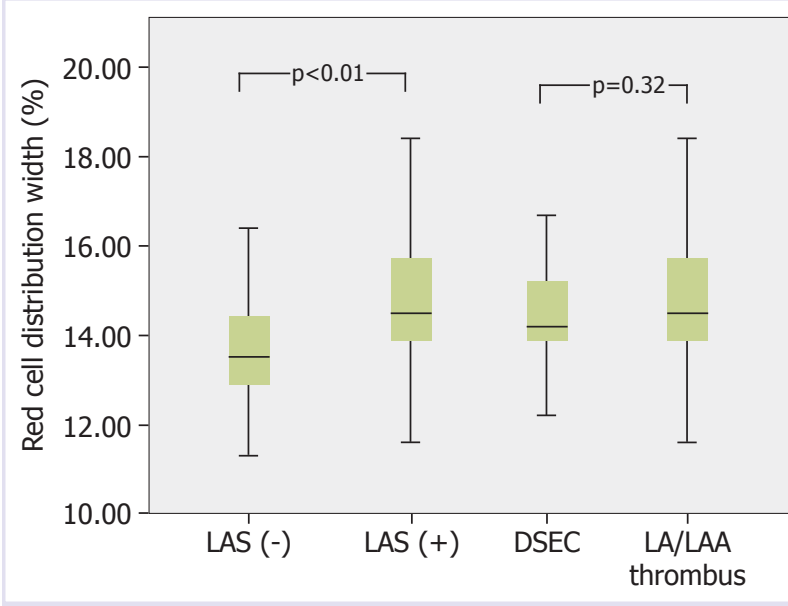

FIGURE 1. Box-plot graph demonstrating comparison of red cell distribution width level between the study groups. DSEC: Dense spontaneous echo contrast; LA: Left atrium; LAA: Left atrial appendage; LAS: Left atrial stasis. 


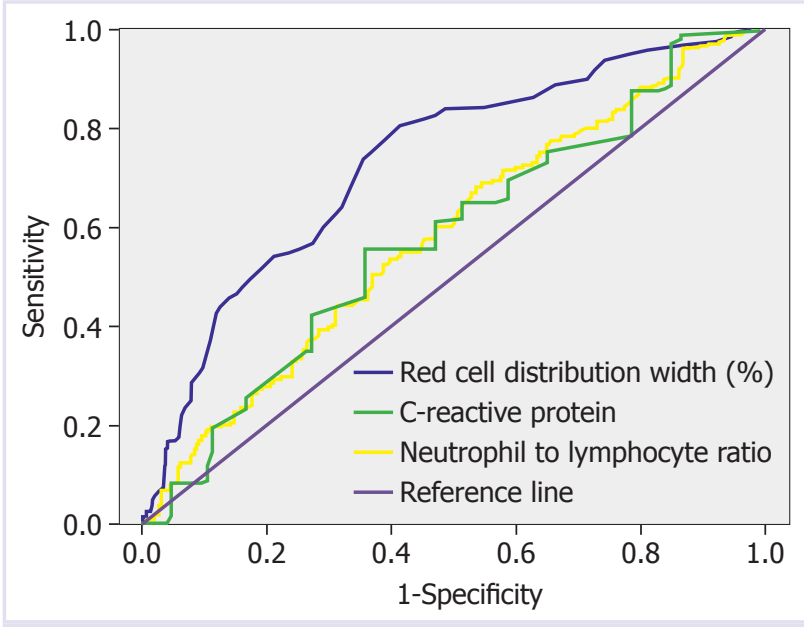

FIGURE 2. Receiver operating characteristic curve analysis of red cell distribution width, neutrophil to lymphocyte ratio, and high sensitivity C-reactive protein level for prediction of left atrial stasis in the study population.

RDW $>13.7 \%$ predicted presence of LAS with sensitivity of $78.1 \%$ and specificity of $58.5 \%$. In patients with RDW $>13.7 \%$, rate of LAS (+) was $68.9 \%$, which was significantly higher than in patients with RDW $\leq 13.7 \%$ (30.6\%) ( $<<0.01)$.

Univariate and multivariate binary logistic regression analysis was performed to investigate inde- pendent correlates of LAS in the study population. In multivariable model, age, male gender, heart failure, AF duration $>6$ months, and increased RDW level (odds ratio, 1.67; 95\% confidence interval, 1.44-1.94; $\mathrm{p}<0.01)$ were found to be independently correlated with presence of LAS (Table 3).

\section{DISCUSSION}

In this study, we determined correlation between increased RDW level and increased risk for LA/LAA thrombus formation and DSEC in Turkish patients with non-valvular AF. In multivariate binary logistic regression analysis, older age, male gender, heart failure, AF duration $>6$ months, and increased RDW level were found to be independently correlated with presence of LAS. NLR was determined to be significantly higher in study group, but it was not an independent predictor when combined with clinical risk factors. To the best of our knowledge, this is the first study in which RDW independently predicted LAS.

$\mathrm{AF}$ is the most common sustained tachyarrhythmia and is associated with increased cardiovascular morbidity, mortality, and preventable stroke, accounting for approximately one-third of cardiac hospitalizations for cardiac rhythm disturbances [12].

TABLE 3. Univariate and multivariate regression analysis for predictors of left atrial stasis in the study population

\begin{tabular}{lcccc} 
Variables & Unadjusted OR (95\% CI) & $\mathrm{p}$ & Adjusted OR (95\% CI) & $\mathrm{p}$ \\
\hline Age & $1.05(1.03-1.06)$ & $<0.01$ & $1.03(1.01-1.05)$ & $<0.01$ \\
Male gender & $1.42(1.03-1.96)$ & 0.03 & $1.53(1.05-2.31)$ & 0.04 \\
Hypertension & $2.52(1.79-3.56)$ & $<0.01$ & $0.98(0.61-1.58)$ & 0.92 \\
Heart failure & $5.30(2.79-10.1)$ & $<0.01$ & $3.59(1.68-6.86)$ & $<0.01$ \\
Diabetes mellitus & $2.09(1.23-3.51)$ & $<0.01$ & $1.09(0.59-2.11)$ & 0.77 \\
AF duration $>$ 6 months & $6.29(4.28-9.24)$ & $<0.01$ & $3.25(2.08-5.12)$ & $<0.01$ \\
Creatinine & $2.80(1.44-5.48)$ & $<0.01$ & $1.41(0.63-3.45)$ & 0.75 \\
CRP* & $1.32(1.024-1.69)$ & 0.03 & - & - \\
Hemoglobin & $0.91(0.81-1.01)$ & 0.11 & - & - \\
RDW & $1.81(1.57-2.08)$ & $<0.01$ & $1.67(1.44-1.94)$ & $<0.01$ \\
NLR & $1.08(0.99-1.17)$ & 0.06 & $0.98(0.89-1.07)$ & 0.61 \\
\hline
\end{tabular}

AF: Atrial fibrillation; CI: Confidence interval; CRP: C-reactive protein; INR: International normalized ratio; OR: Odds ratio; RDW: Red cell distribution width; NLR: Neutrophil to lymphocyte ratio. ${ }^{*}$ CRP level was not included in the multivariable model because this parameter could be obtained in only 524 patients. 
Left atrial stasis, dyssynchrony, and compromised contraction and relaxation are the underlying pathologies in AF that cause SEC, sludge, and thrombus in LA/LAA. Consequently, any thrombus migration from LA/LAA interrupts blood flow of the organ it occludes, and associated disabilities occur.

There may be some concerns about the number and ratio of patients with LAS in our study (325/619), which is unlike 2 previously published papers. There were 88 patients with LAS of 247 patients who underwent TEE in study conducted by Providencia et al. [13]. The other study included 24 patients with LAS of 90 patients who had undergone TEE [14]. Both studies compromised all consecutive TEE patients, while we selected 325 consecutive patients with LAS and matched them with 294 consecutive patients without LAS.

In our study, we found that patients with LAS were older, as well as more often hypertensive, and diabetic. Similarly, they had CHF, vascular disease and persistent AF more often than control group. $\mathrm{CHADS}_{2}$ score, $\mathrm{CHA}_{2} \mathrm{DS}_{2}-\mathrm{VASc}$ score, and warfarin usage were also higher than seen in controls. These demographic properties of the groups were compatible with current literature. However, there were no statistical differences in previous stroke, despite high rate of warfarin use in LAS (+) group. We think patients under warfarin treatment may not have had enough time in therapeutic range, which means ineffective treatment. Zhao et al. [14] found increased warfarin usage in LAS group, as seen in our study, but found that previous stroke was also higher in LAS group, unlike our results.

RDW, which has institutionally been used for differential diagnosis of anemia, is a quantitative measure of variability in the size of circulating erythrocytes, and is now accepted as prognostic index for cardiovascular diseases. It is associated with increased mortality in patients with stroke, $\mathrm{CHF}$, and myocardial infarction; however, there is no clear pathophysiological mechanism yet for this association. Chronic inflammation and oxidative stress, which are present in both development and persistence of AF, may reduce number of red blood cells, which in turn increases erythropoietin production and leads to increase in RDW. Higher RDW level was associated with high level of CRP, erythropoietin, interleukin-6, tumor necrosis factor (TNF) alpha, and TNF receptor I/II in systolic heart failure patients [15], which revealed association of chronic inflammation with RDW level.

Association of RDW and non-valvular AF has recently been reported [16]. There are 2 studies that investigated association of RDW and LAS in nonvalvular $A F$, and both failed to show independent correlation of RDW with LAS. The study conducted by Providencia et al. [13] indicated that RDW and mean corpuscular volume (MCV) add incremental value to each other, but when clinical risk factors were added, MCV remains independent predictor. Zhao et al. [14] also reported association of RDW with LAS, but when clinical risk factors were added, low hemoglobin and persistence of AF remained independent predictors. In our study, even after adding clinical risk factors, RDW was independent predictor of LAS.

Increased neutrophil count is consistent with subclinical inflammation, while decreased lymphocyte count shows physiological stress and poor general health [17]. This means NLR could be used as a monitor for inflammatory status and stress response. In this study, even though NLR was higher in LAS (+) group, we did not observe independent correlation between NLR and presence of LAS $(+)$ when RDW levels and clinical factors were included in multivariable regression model. Chronic inflammation could have a role in development of AF. It generates electrical and structural remodeling, forming substrate for development and persistence of arrhythmia. Ongoing rhythm disturbance with atrial endothelial destruction due to inflammation results in thrombus formation in LAA. In other words, severity of inflammatory status in LA may be correlated with risk of LA/LAA thrombus formation.

\section{Limitations}

There are some potential limitations with the present study. First of all, this was a retrospective study and demonstrates association of variables rather than cause-effect relationship. In addition, our findings do not describe clinical use of $\mathrm{CBC}$ parameters 
in prediction of future thromboembolic events in patients with AF. Lack of parameters, especially iron/ferritin level, can be accepted as a limitation. Lack of parameters demonstrating inflammation other than CRP could also be considered a limitation of our study. Rate of LA/LAA thrombus formation was higher than seen in previous studies 13,14 . This finding may suggest that effectiveness and duration of anticoagulation was not adequate for resolution of thrombi at time of TEE examination. For further investigation of this topic, multicenter, prospective studies are warranted.

\section{Conclusion}

RDW is independent predictor of LAS in non-valvular AF in Turkish population. Larger prospective studies may reveal clinical use of $\mathrm{CBC}$ parameters, and especially RDW level, for defining cases with higher risk of LAS, embolization, and cerebrovascular events.

\section{Conflict of Interest: None declared.}

Financial Disclosure: The authors declared that this study has received no financial support.

Authorship contributions: Concept - A.K., A.T.A., C.T., A.I.T; Design - A.K., B.G., K.S.O., M.A.T., O.K.; Supervision O.K., G.G., F.O.K., M.A.T.; Materials - B.G., A.K., G.G., M.A.T., C.T., A.I.T., F.O.K.; Data collection \&/or processing - A.K., C.T., A.I.T., A.T.A., F.O.K., G.G.; Analysis and/or interpretation - G.G., O.K., B.G., K.S.O., A.I.T.; Literature search - K.S.O., M.A.T., G.G., F.O.K., A.T.A., C.T.; Writing - A.K., B.G.; Critical review - A.T.A., B.G., A.I.T., O.K., K.S.O.

\section{REFERENCES}

1. Morady F, Zipes DP. Atrial fibrillation: clinical features, mechanisms and management. In: Braunwald E, Libby P, Bonow RO, Mann DL, Zipes DP, editors. Braunwald's heart disease. A textbook of cardiovascular medicine. $9^{\text {th }}$ ed. Philadelphia, PA: WB Saunders Company; 2012. p. 825-84.

2. Khoo CW, Krishnamoorthy S, Lim HS, Lip GY. Atrial fibrillation, arrhythmia burden and thrombogenesis. Int J Cardiol 2012;157:318-23.

3. Watson T, Shantsila E, Lip GY. Mechanisms of thrombogenesis in atrial fibrillation: Virchow's triad revisited. Lancet 2009;373:155-66.

4. Lappé JM, Horne BD, Shah SH, May HT, Muhlestein JB, Lappé DL, et al. Red cell distribution width, C-reactive protein, the complete blood count, and mortality in patients with coronary disease and a normal comparison population. Clin Chim Acta
2011;412:2094-9.

5. Felker GM, Allen LA, Pocock SJ, Shaw LK, McMurray JJ, Pfeffer MA, et al; CHARM Investigators. Red cell distribution width as a novel prognostic marker in heart failure: data from the CHARM Program and the Duke Databank. J Am Coll Cardiol 2007;50:40-7.

6. Tonelli M, Sacks F, Arnold M, Moye L, Davis B, Pfeffer M; for the Cholesterol and Recurrent Events (CARE) Trial Investigators. Relation Between Red Blood Cell Distribution Width and Cardiovascular Event Rate in People With Coronary Disease. Circulation 2008;117:163-8.

7. Klein AL, Grimm RA, Murray RD, Apperson-Hansen C, Asinger RW, Black IW, et al; Assessment of Cardioversion Using Transesophageal Echocardiography Investigators. Use of transesophageal echocardiography to guide cardioversion in patients with atrial fibrillation. N Engl J Med 2001;344:1411-20.

8. Scherr D, Dalal D, Chilukuri K, Dong J, Spragg D, Henrikson $\mathrm{CA}$, et al. Incidence and predictors of left atrial thrombus prior to catheter ablation of atrial fibrillation. J Cardiovasc Electrophysiol 2009;20:379-84.

9. Kerut EK, Hanawalt C, McKinnie J. Transesophageal echocardiography during pulmonary vein cryoballoon ablation for atrial fibrillation. Echocardiography 2015;32:281-90.

10. Fatkin D, Loupas T, Jacobs N, Feneley MP. Quantification of blood echogenicity: evaluation of a semiquantitative method of grading spontaneous echo contrast. Ultrasound Med Biol 1995;21:1191-8.

11. Olesen JB, Lip GY, Hansen ML, Hansen PR, Tolstrup JS, Lindhardsen J, et al. Validation of risk stratification schemes for predicting stroke and thromboembolism in patients with atrial fibrillation: nationwide cohort study. BMJ 2011;342:d124.

12. Griffin BP. Manual of cardiovascular medicine. 13 th edition. Philadelphia PA: Lippincott Williams and Wilkins; 2013. p. 424.

13. Providência R, Ferreira MJ, Gonçalves L, Faustino A, Paiva L, Fernandes A, et al. Mean corpuscular volume and red cell distribution width as predictors of left atrial stasis in patients with non-valvular atrial fibrillation. Am J Cardiovasc Dis 2013;3:91102.

14. Zhao J, Liu T, Korantzopoulos P, Fu H, Shao Q, Suo Y, et al. Red blood cell distribution width and left atrial thrombus or spontaneous echo contrast in patients with non-valvular atrial fibrillation. Int J Cardiol 2015;180:63-5.

15. Förhécz Z, Gombos T, Borgulya G, Pozsonyi Z, Prohászka Z, Jánoskuti L. Red cell distribution width in heart failure: prediction of clinical events and relationship with markers of ineffective erythropoiesis, inflammation, renal function, and nutritional state. Am Heart J 2009;158:659-66.

16. Güngör B, Özcan KS, Erdinler İ, Ekmekçi A, Alper AT, Osmonov D, et al. Elevated levels of RDW is associated with nonvalvular atrial fibrillation. J Thromb Thrombolysis 2014;37:40410.

17. Canpolat U, Aytemir K, Yorgun H, Şahiner L, Kaya EB, Kabakçı $\mathrm{G}$, et al. Role of preablation neutrophil/lymphocyte ratio on outcomes of cryoballoon-based atrial fibrillation ablation. Am J Cardiol 2013;112:513-9. 